\title{
A UHF micro-power CMOS rectifier using a novel diode connected CMOS transistor for micro-sensor and RFID applications
}

\begin{abstract}
The design strategy and efficiency optimization of UHF micro-power rectifiers using a novel diode connected MOS transistor is presented. The proposed diode connected MOS transistor uses a new bulk connection which leads to reduce the threshold voltage and leakage current in compare to conventional diode connected transistors. Using the proposed diode in typical rectifiers makes a significant improvement in output voltage and current therefore the efficiency is increased comparing to the same rectifier architectures using conventional diodes. Also a design procedure for efficiency optimization is presented and a superposition method is used to optimize the performance of multiple output rectifiers. Finally a five stage double output rectifier is designed based on the proposed optimization method. Simulation results verified the improvement in efficiency and area. All circuits are designed in a standard 0.18um CMOS Technology.
\end{abstract}

Keyword: RF energy harvesting; CMOS rectifier; Diode connected CMOS transistor; Efficiency; Super position 